\title{
Observability Analysis of a Vision-aided Inertial Navigation System Using Planar Features on the Ground
}

\author{
Ghazaleh Panahandeh, Chao X. Guo, Magnus Jansson, and Stergios I. Roumeliotis
}

\begin{abstract}
In this paper, we present an observability analysis of a vision-aided inertial navigation system (VINS) in which the camera is downward looking and observes a single point feature on the ground. In our analysis, the full INS parameter vector (including position, velocity, rotation, and inertial sensor biases) as well as the $3 \mathrm{D}$ position of the observed point feature are considered as state variables. In particular, we prove that the system has only three unobservable directions corresponding to global translations along the $x$ and $y$ axes, and rotations around the gravity vector. Hence, compared to general VINS, an advantage of using only ground features is that the vertical translation becomes observable. The findings of the theoretical analysis are validated through real-world experiments.
\end{abstract}

\section{INTRODUCTION}

In this paper, we are interested in studying the observability properties of an inertial measurement unit (IMU)-camera sensor fusion system, which is navigating in an unknown environment. In particular, our analysis is performed for a downward looking camera (DLC), which observes planar features on the ground, i.e., the image plane is parallel to the feature's plane. Such a configuration may find interesting applications in different scenarios. For example, where there are many moving objects (such as pedestrians and vehicles) in front of the camera, a forward-looking camera may not be able to detect and reliably track static features, while, a DLC can still track point features on the ground [1]. Furthermore, in certain cases, such as micro air vehicle (MAV) or spacecraft landing [2], most of the features observed by the DLC lie on the ground, and one should take advantage of this fact to improve the estimation accuracy.

The observability properties of the general vision-aided inertial navigation system (VINS)-without using the assumption of observing features in a plane-have been thoroughly studied in the literature. Specifically in [3], [4], the authors prove that all the quantities of the IMU-camera sensor fusion system (i.e., the robot pose, velocity, IMU biases, feature positions and the transformation between the IMU and the camera) are observable given observations of known point features [3], or when navigating from a known initial pose [4]. Without these assumptions, it has been shown

Ghazaleh Panahandeh and Magnus Jansson are with the ACCESS Linnaeus Centre, School of Electrical Engineering, KTH Royal Institute of Technology, SE-100 44 Stockholm, Sweden.

\{ghpa, janssonm\}akth.se

Chao X. Guo and Stergios I. Roumeliotis are with the Department of Computer Science and Engineering, University of Minnesota, Minneapolis, MN 55455, USA. \{chaguo, stergios\}@cs.umn.edu.

This work was supported by the Swedish Research Council (VR), the Swedish Governmental Agency for Innovation Systems (VINNOVA), and by the University of Minnesota through the Digital Technology Center (DTC) and AFOSR (FA9550-10-1-0567). that the general VINS is unobservable, and its unobservable directions are the global position, as well as rotation around the gravity vector [5], [6]. In [7], the observability properties of VINS has been examined for different sensor configurations including: inertial only, vision only, vision and inertial. In [8] the concept of continuous symmetries is used for analyzing the observability properties of VINS and it is shown that the IMU biases, 3D velocity, and absolute roll and pitch angles, are observable for VINS. To the best of our knowledge, the only work that studies the observability analysis of a VINS when observing a feature in a horizontal plane is the work of [9]. However, since a virtual feature projected from a laser pointer mounted on the robot is tracked, the analysis is performed for a special camera measurement model. Additionally, in [9] the biases in the IMU measurements are ignored both in the observability analysis and in the estimator, which can result in significant drift.

To the best of our knowledge, the work presented in this paper is the first to examine the observability properties of the DLC-VINS when observing ground point features. In our observability analysis, we employ the method introduced in [10], which significantly reduces the complexity of finding the system's unobservable directions (Section II). In particular, in Section IV, we prove that by observing a single unknown feature on the ground, the navigation system has only three unobservable directions corresponding to the global translations parallel to the ground plane, and the rotation around the gravity vector. Finally, in Section V, we experimentally illustrate the validity of our analysis using the method introduced in [1].

\section{NONLINEAR OBSERVABILITy ANALySiS}

In what follows, we first provide an overview of the nonlinear observability rank condition test of [11] (Section II-A) and summarize the method of [10] for finding the unobservable modes of our system (Section II-B).

\section{A. Observability Analysis with Lie Derivatives}

Consider a nonlinear system ${ }^{1}$

$$
\left\{\begin{array}{l}
\dot{\mathbf{x}}=\mathbf{f}_{0}(\mathbf{x})+\sum_{i=1}^{\ell} \mathbf{f}_{i}(\mathbf{x}) u_{i} \\
\mathbf{z}=\mathbf{h}(\mathbf{x})
\end{array}\right.
$$

where $\mathbf{x} \in \mathbb{R}^{m}$ is the state vector, $\mathbf{u}=\left[\begin{array}{lll}u_{1} & \ldots & u_{\ell}\end{array}\right]^{\top} \in \mathbb{R}^{\ell}$ is the system input, $\mathbf{z} \in \mathbb{R}^{k}$ is the system output, and $\mathbf{f}_{i}$ for $i \in\{0, \ldots, \ell\}$ is the process function.

\footnotetext{
${ }^{1}$ Throughout the paper, scalars are denoted by lowercase letters $(s)$, vectors by bold letters (f), and matrices by bold capitals $(\mathbf{K})$.
} 
The zeroth order Lie derivative of a measurement function $\mathbf{h}$ is the function itself, i.e., $\mathfrak{L}^{0} \mathbf{h}=\mathbf{h}(\mathbf{x})$. For any $n$-th order Lie derivative, $\mathfrak{L}^{n} \mathbf{h}$, the $n+1$-th order Lie derivative $\mathfrak{L}_{\mathbf{f}_{i}}^{n+1} \mathbf{h}$ with respect to a process function $\mathbf{f}_{i}$ can be computed as:

$$
\mathfrak{L}_{\mathbf{f}_{i}}^{n+1} \mathbf{h}=\nabla \mathfrak{L}^{n} \mathbf{h} \cdot \mathbf{f}_{i},
$$

where $\nabla$ denotes the gradient operator with respect to $\mathbf{x}$ and "." represents the vector inner product. Similarly, mixed higher order Lie derivatives can be defined as:

$$
\mathfrak{L}_{\mathbf{f}_{i} \mathbf{f}_{j} \ldots \mathbf{f}_{k}}^{n} \mathbf{h}=\mathfrak{L}_{\mathbf{f}_{i}}\left(\mathfrak{L}_{\mathbf{f}_{j} \ldots \mathbf{f}_{k}}^{n-1} \mathbf{h}\right)=\nabla \mathfrak{L}_{\mathbf{f}_{j} \ldots \mathbf{f}_{k}}^{n-1} \mathbf{h} \cdot \mathbf{f}_{i}
$$

where $i, j, k \in\{0, \ldots, \ell\}$. The observability of a system is determined by calculating the dimension of the space spanned by the gradients of the Lie derivatives of its output functions [11]. Hence, the observability matrix $\mathbf{O}$ of system (1) is defined as:

$$
\mathbf{O} \triangleq\left[\begin{array}{c}
\nabla \mathfrak{L}^{0} \mathbf{h} \\
\nabla \mathfrak{L}_{\mathbf{f}_{i}}^{1} \mathbf{h} \\
\vdots \\
\nabla \mathfrak{L}_{\mathbf{f}_{i} \mathbf{f}_{j} \ldots \mathbf{f}_{k}}^{n} \mathbf{h} \\
\vdots
\end{array}\right] .
$$

To prove that a system is observable, it suffices to show that $\mathbf{O}$ is of full column rank. However, to prove that a system is unobservable, we have to find the null space of matrix $\mathbf{O}$, which may have infinitely many rows. This can be very challenging especially for high-dimensional systems, such as the DLC-VINS. To address this issue, in the following section we present the method of [10] for proving that a system is unobservable and finding its unobservable directions.

\section{B. Observability Analysis with Basis Functions}

Theorem 1: Assume that there exists a nonlinear transformation $\boldsymbol{\beta}(\mathbf{x})=\left[\boldsymbol{\beta}_{1}(\mathbf{x})^{\top}, \ldots, \boldsymbol{\beta}_{n}(\mathbf{x})^{\top}\right]^{\top}$ (i.e., a set of basis functions) of the variable $\mathbf{x}$, such that:

1) The system measurement equation can be written as a function of $\boldsymbol{\beta}$, i.e., $\mathbf{z}=\mathbf{h}(\mathbf{x})=\overline{\mathbf{h}}(\boldsymbol{\beta})$

2) $\frac{\partial \boldsymbol{\beta}}{\partial \mathbf{x}} \mathbf{f}_{j}$, for $j=0, \ldots, \ell$, is a function of $\boldsymbol{\beta}$

Then the observability matrix of system (1) can be factorized as: $\mathbf{O}=\Xi \Omega$ where $\Xi$ is the observability matrix of the system

$$
\left\{\begin{array}{l}
\dot{\boldsymbol{\beta}}=\mathbf{g}_{0}(\boldsymbol{\beta})+\sum_{i=1}^{\ell} \mathbf{g}_{i}(\boldsymbol{\beta}) u_{i} \\
\mathbf{z}=\overline{\mathbf{h}}(\boldsymbol{\beta})
\end{array}\right.
$$

and $\Omega \triangleq \frac{\partial \boldsymbol{\beta}}{\partial \mathbf{x}}$.

Proof: See [10].

Note that system (5) results by pre-multiplying the process function in (1) with $\frac{\partial \boldsymbol{\beta}}{\partial \mathbf{x}}$

$$
\begin{aligned}
&\left\{\begin{array}{l}
\frac{\partial \boldsymbol{\beta}}{\partial \mathbf{x}} \frac{\partial \mathbf{x}}{\partial t}=\frac{\partial \boldsymbol{\beta}}{\partial \mathbf{x}} \mathbf{f}_{0}(\mathbf{x})+\frac{\partial \boldsymbol{\beta}}{\partial \mathbf{x}} \sum_{i=1}^{\ell} \mathbf{f}_{i}(\mathbf{x}) u_{i} \\
\mathbf{z}=\mathbf{h}(\mathbf{x})
\end{array}\right. \\
& \Rightarrow \quad\left\{\begin{array}{l}
\dot{\boldsymbol{\beta}}=\mathbf{g}_{0}(\boldsymbol{\beta})+\sum_{i=1}^{\ell} \mathbf{g}_{i}(\boldsymbol{\beta}) u_{i} \\
\mathbf{z}=\overline{\mathbf{h}}(\boldsymbol{\beta})
\end{array}\right.
\end{aligned}
$$

where $\mathbf{g}_{i}(\boldsymbol{\beta}) \triangleq \frac{\partial \boldsymbol{\beta}}{\partial \mathbf{x}} \mathbf{f}_{i}(\mathbf{x})$ and $\overline{\mathbf{h}}(\boldsymbol{\beta}) \triangleq \mathbf{h}(\mathbf{x})$.
Corollary 1: If $\Xi$ is of full column rank, i.e., system (5) is observable, then the unobservable directions of system (1) will be spanned by the null vectors of $\Omega$.

Proof: From $\mathbf{O}=\Xi \Omega$, we have $\operatorname{null}(\mathbf{O})=\operatorname{null}(\Omega) \cup$ $(\operatorname{null}(\Xi) \cap \operatorname{range}(\Omega))$. Therefore, if $\Xi$ is of full column rank, i.e., system (5) is observable, then $\operatorname{null}(\mathbf{O})=\operatorname{null}(\Omega)$.

Based on Theorem 1 and Corollary 1, to find the unobservable directions of a system, we first need to define the basis functions, $\boldsymbol{\beta}$, which fulfil the first and second conditions of Theorem 1. Then, we should prove that the infinite-dimensional matrix $\Xi$ has full column rank, which satisfies the condition of Corollary 1 .

To define the basis functions, we start with the system measurement equation and extract the initial bases as a function of the state variables (i.e., the first condition of Theorem 1). The rest of the bases will be defined by projecting these initial basis functions onto the process functions. Then, any resulting term that cannot be expressed as a function of the previously defined bases, is incorporated as a new basis function (i.e., the second condition of the Theorem 1). Finally, we terminate the procedure of defining new basis functions when the second condition of Theorem 1 is satisfied.

\section{SySTEM MODEL}

Before studying the observability properties of our system in Section IV, we hereafter describe its process and measurement equations.

For the purpose of IMU-camera ego-motion estimation, we consider the sensors to be rigidly attached to a moving vehicle. The goal is to estimate the pose of this mobile VINS with respect to the global frame $\{G\}$. In this system, the IMU measurements are used for state propagation while the camera measurements are employed for state corrections.

1) System propagation model: We define the INS state variables in the form of a system state vector as: ${ }^{2}$

$$
\mathbf{x}=\left[\begin{array}{lllll}
{ }^{C} \mathbf{S}_{G}^{\top} & { }^{G} \mathbf{v}_{C}^{\top} & { }^{G} \mathbf{p}_{C}^{\top} & \mathbf{b}_{a}^{\top} & \mathbf{b}_{g}^{\top}
\end{array}\right]^{\top}
$$

where ${ }^{C} \mathbf{S}_{G}$ is the Cayley-Gibbs-Rodriguez parameterization [12] representing the orientation of the global frame $\{G\}$ in the camera's frame of reference $\{C\},{ }^{G} \mathbf{v}_{C}$ and ${ }^{G} \mathbf{p}_{C}$ denote the velocity and position of $\{C\}$ in $\{G\}$, respectively, and $\mathbf{b}_{a}$ and $\mathbf{b}_{g}$ are the biases in the gyroscope and accelerometer measurements. The system model describing the time evolution of the VINS states is:

$$
\begin{aligned}
& { }^{C} \dot{\mathbf{S}}_{G}(t)=\frac{1}{2} \mathbf{D}\left({ }^{C} \boldsymbol{\omega}(t)-\mathbf{b}_{g}(t)\right) \\
& { }^{G} \dot{\mathbf{v}}_{C}(t)={ }^{G} \mathbf{a}(t)={ }^{G} \mathbf{g}+\mathbf{C}\left({ }^{C} \mathbf{s}_{G}(t)\right)^{\top}\left({ }^{C} \mathbf{a}(t)-\mathbf{b}_{a}(t)\right) \\
& { }^{G} \dot{\mathbf{p}}_{C}(t)={ }^{G} \mathbf{v}_{C}(t) \quad \dot{\mathbf{b}}_{a}(t)=\mathbf{n}_{a} \quad \dot{\mathbf{b}}_{g}(t)=\mathbf{n}_{g}
\end{aligned}
$$

where $^{3} \frac{1}{2} \mathbf{D} \triangleq \frac{\partial \mathbf{s}}{\partial \boldsymbol{\theta}}=\frac{1}{2}\left(\mathbf{I}+\lfloor\mathbf{s}\rfloor+\mathbf{s} \mathbf{S}^{\top}\right) \quad(\boldsymbol{\theta}=\alpha \hat{\mathbf{k}}$ represents a rotation by an angle $\alpha$ around the axis $\hat{\mathbf{k}}),{ }^{C} \boldsymbol{\omega}(t)=$

\footnotetext{
${ }^{2}$ To preserve the clarity of the presentation, we assume that the IMU and camera frames of reference coincide.

${ }^{3}$ The skew-symmetric matrix of vector $\mathbf{a}$ is represented by $\lfloor\mathbf{a}\rfloor$. Throughout this paper, the following properties of the cross product and the skew-symmetric matrix operator $(\lfloor\mathbf{a}\rfloor)$ are used: $\mathbf{a} \times \mathbf{b}=\lfloor\mathbf{a}\rfloor \mathbf{b}=-\lfloor\mathbf{b}\rfloor \mathbf{a}$, $\lfloor\mathbf{a}\rfloor \mathbf{a}=\mathbf{0}$, and $\lfloor\mathbf{a}\rfloor\lfloor\mathbf{b}\rfloor=\mathbf{b a}^{\top}-\left(\mathbf{a}^{\top} \mathbf{b}\right) \mathbf{I}_{3}$.
} 
$\left[\begin{array}{lll}\omega_{1} & \omega_{2} & \omega_{3}\end{array}\right]^{\top}$ and ${ }^{C} \mathbf{a}(t)=\left[\begin{array}{lll}a_{1} & a_{2} & a_{3}\end{array}\right]^{\top}$ are the rotational velocity and linear acceleration, respectively, measured by the IMU and expressed in $\{C\} .{ }^{G} \mathbf{g}=\left[\begin{array}{lll}0 & 0 & g\end{array}\right]^{\top}$ is the gravitational acceleration, $\mathbf{C}(\mathbf{s})$ is the rotation matrix corresponding to $\mathbf{s}$, and $\mathbf{n}_{g}$ and $\mathbf{n}_{a}$ are the accelerometer and gyroscope bias driving white Gaussian noises.

2) System measurement model: Assuming a calibrated pinhole camera, the projective camera measurement model is 4

$$
\mathbf{z}^{C}=\left[\begin{array}{c}
u \\
v
\end{array}\right]+\mathbf{n}_{\mathbf{z}}=\mathbf{B} \frac{1}{\mathbf{e}_{3}^{\top C} \mathbf{p}_{f}}{ }^{C} \mathbf{p}_{f}+\mathbf{n}_{\mathbf{z}}
$$

where

$$
{ }^{C} \mathbf{p}_{f}=\mathbf{C}\left({ }^{C} \mathbf{s}_{G}\right)\left({ }^{G} \mathbf{p}_{f}-{ }^{G} \mathbf{p}_{C}\right) \text { and } \mathbf{B}=\left[\begin{array}{ll}
\mathbf{I}_{2} & 0_{2 \times 1}
\end{array}\right],
$$

${ }^{C} \mathbf{p}_{f}$ and ${ }^{G} \mathbf{p}_{f}$ represent the position of the feature point $f$ with respect to the camera and global coordinate frame, respectively, and $\mathbf{n}_{\mathbf{z}}$ is the image pixel noise.

Without loss of generality, the global frame is considered to be on the ground plane with its $z$ axis pointing up. Additionally, in our DLC-VINS, it is assumed that the camera's optical axis is orthogonal to the ground plane and the point features lie on the ground. ${ }^{5}$ Thus, $\mathbf{e}_{3}^{\top C} \mathbf{p}_{f}=\mathbf{e}_{3}^{\top G} \mathbf{p}_{C}$ and the camera measurement model can be rewritten as:

$$
\mathbf{z}^{C}=\mathbf{B} \frac{1}{\mathbf{e}_{3}^{\top}{ }^{G} \mathbf{p}_{C}}{ }^{C} \mathbf{p}_{f}+\mathbf{n}_{\mathbf{z}} .
$$

Moreover, this particular geometric configuration implies a constraint on the velocity along the $z$ axis which can be represented as an additional measurement

$$
\mathbf{e}_{3}^{\top} \mathbf{C}^{G} \mathbf{v}_{C}=\mathbf{e}_{3}^{\top G} \mathbf{v}_{C} \quad \Leftrightarrow \quad z^{v}=\mathbf{e}_{3}^{\top} \mathbf{C}^{G} \mathbf{v}_{C}-\mathbf{e}_{3}^{\top G} \mathbf{v}_{C}=0 .
$$

\section{System Observability AnAlysis}

In what follows, we first define the basis functions for the DLC-VINS (Section IV-A) and then derive its unobservable modes (Section IV-B).

\section{A. Defining the Basis Functions}

In our analysis, the position of the observed point feature with respect to the global coordinate frame, ${ }^{G} \mathbf{p}_{f}$ is considered as an unknown constant variable

$$
{ }^{G} \dot{\mathbf{p}}_{f}=\mathbf{0},
$$

which we append to the state vector (see [5]).

For simplicity, we retain only a few of the subscripts and superscripts in the state elements and write the augmented DLC-INS state vector (6) as:

$$
\mathbf{x}=\left[\begin{array}{llllll}
\mathbf{s}^{\top} & \mathbf{v}^{\top} & \mathbf{p}^{\top} & \mathbf{b}_{a}^{\top} & \mathbf{b}_{g}^{\top} & \mathbf{p}_{f}^{\top}
\end{array}\right]^{\top} .
$$

$$
[0,0,1] \text {. }
$$

${ }^{4} \mathbf{e}_{i} \in \mathbb{R}^{3 \times 1}$ for $i=1,2,3$ and $\mathbf{e}_{1}^{\top}=[1,0,0], \mathbf{e}_{2}^{\top}=[0,1,0]$, and $\mathbf{e}_{3}^{\top}=$

${ }^{5}$ Although the assumption of the orthogonality of the camera's optical axis to the ground leads to some nice properties, this assumption might be violated in a real scenario. This constraint is relaxed in [1] using the virtual camera concept.
Moreover, following the structure of system (1), we rewrite the state propagation equation in (7) and (11) as

$$
\left[\begin{array}{c}
\dot{\mathbf{s}} \\
\dot{\mathbf{v}} \\
\dot{\mathbf{p}} \\
\dot{\mathbf{b}}_{a} \\
\dot{\mathbf{b}}_{g} \\
\dot{\mathbf{p}}_{f}
\end{array}\right]=\underbrace{\left[\begin{array}{c}
-\frac{1}{2} \mathbf{D b}_{g} \\
\mathbf{g}-\mathbf{C}^{T} \mathbf{b}_{a} \\
\mathbf{v} \\
\mathbf{0} \\
\mathbf{0} \\
\mathbf{0}
\end{array}\right]}_{\mathbf{f}_{0}}+\underbrace{\left[\begin{array}{c}
\frac{1}{2} \mathbf{D} \\
\mathbf{0} \\
\mathbf{0} \\
\mathbf{0} \\
\mathbf{0} \\
\mathbf{0}
\end{array}\right]}_{\mathbf{F}_{1}} \boldsymbol{\omega}+\underbrace{\left[\begin{array}{c}
\mathbf{0} \\
\mathbf{C}^{T} \\
\mathbf{0} \\
\mathbf{0} \\
\mathbf{0} \\
\mathbf{0}
\end{array}\right]}_{\mathbf{F}_{2}} \mathbf{a}
$$

where $\mathbf{C} \triangleq \mathbf{C}(\mathbf{s})$. Note that $\mathbf{f}_{0}$ is a $24 \times 1$ vector, while $\mathbf{F}_{1}$ and $\mathbf{F}_{2}$ are both $24 \times 3$ matrices which is a compact form for representing three process functions as

$$
\begin{aligned}
\mathbf{F}_{1} \boldsymbol{\omega} & =\mathbf{f}_{11} \omega_{1}+\mathbf{f}_{12} \omega_{2}+\mathbf{f}_{13} \omega_{3} \\
\mathbf{F}_{2} \mathbf{a} & =\mathbf{f}_{21} a_{1}+\mathbf{f}_{22} a_{2}+\mathbf{f}_{23} a_{3}
\end{aligned}
$$

Following the first condition of Theorem 1, we define the system's first four bases using the terms appearing in the measurement functions (9) and (10), i.e.,

$$
\boldsymbol{\beta}_{1} \triangleq \mathbf{C}\left(\mathbf{p}_{f}-\mathbf{p}\right), \quad \boldsymbol{\beta}_{2} \triangleq \mathbf{e}_{3}^{\top} \mathbf{p}, \quad \boldsymbol{\beta}_{3} \triangleq \mathbf{C v}, \quad \boldsymbol{\beta}_{4} \triangleq \mathbf{e}_{3}^{\top} \mathbf{v} .
$$

To check the second condition of Theorem 1, we compute their derivatives with respect to the state vector $\mathbf{x}$

$$
\begin{aligned}
\frac{\partial \boldsymbol{\beta}_{1}}{\partial \mathbf{x}} & =\left[\begin{array}{lllllll}
\left\lfloor\mathbf{C}\left(\mathbf{p}_{f}-\mathbf{p}\right)\right\rfloor \frac{\partial \theta}{\partial s} & \mathbf{0} & -\mathbf{C} & \mathbf{0} & \mathbf{0} & \mathbf{C}
\end{array}\right], \\
\frac{\partial \beta_{2}}{\partial \mathbf{x}} & =\left[\begin{array}{llllll}
\mathbf{0} & \mathbf{0} & \mathbf{e}_{3}^{\top} & \mathbf{0} & \mathbf{0} & \mathbf{0}
\end{array}\right], \\
\frac{\partial \boldsymbol{\beta}_{3}}{\partial \mathbf{x}} & =\left[\begin{array}{lllllll}
\lfloor\mathbf{C} \mathbf{v}\rfloor \frac{\partial \theta}{\partial s} & \mathbf{C} & \mathbf{0} & \mathbf{0} & \mathbf{0} & \mathbf{0}
\end{array}\right], \\
\frac{\partial \beta_{4}}{\partial \mathbf{x}} & =\left[\begin{array}{lllllll}
\mathbf{0} & \mathbf{e}_{3}^{\top} & \mathbf{0} & \mathbf{0} & \mathbf{0} & \mathbf{0}
\end{array}\right]
\end{aligned}
$$

and project them onto all the process functions. Specifically, for the span of $\boldsymbol{\beta}_{1} \triangleq \mathbf{C}\left(\mathbf{p}_{f}-\mathbf{p}\right)$, we have:

$$
\begin{aligned}
\frac{\partial \boldsymbol{\beta}_{1}}{\partial \mathbf{x}} \mathbf{f}_{0} & =-\left\lfloor\mathbf{C}\left(\mathbf{p}_{f}-\mathbf{p}\right)\right\rfloor \mathbf{b}_{g}-\mathbf{C v} \\
& =-\left\lfloor\boldsymbol{\beta}_{1}\right\rfloor \mathbf{b}_{g}-\boldsymbol{\beta}_{3} \triangleq-\left\lfloor\boldsymbol{\beta}_{1}\right\rfloor \boldsymbol{\beta}_{5}-\boldsymbol{\beta}_{3} \\
\frac{\partial \boldsymbol{\beta}_{1}}{\partial \mathbf{x}} \mathbf{f}_{1 i} & =\left\lfloor\mathbf{C}\left(\mathbf{p}_{f}-\mathbf{p}\right)\right\rfloor \mathbf{e}_{i}=\left\lfloor\boldsymbol{\beta}_{1}\right\rfloor \mathbf{e}_{i} \\
\frac{\partial \boldsymbol{\beta}_{1}}{\partial \mathbf{x}} \mathbf{f}_{2 i} & =\mathbf{0},
\end{aligned}
$$

for $i \in\{1,2,3\}$, where $\frac{\partial \boldsymbol{\theta}}{\partial \mathbf{s}} \frac{1}{2} \mathbf{D}=\frac{\partial \boldsymbol{\theta}}{\partial \mathbf{s}} \frac{\partial \mathbf{s}}{\partial \boldsymbol{\theta}}=\mathbf{I}_{3}$ and we have defined a new basis element $\boldsymbol{\beta}_{5} \triangleq \mathbf{b}_{g}$. Similarly, for the span of $\beta_{2} \triangleq \mathbf{e}_{3}^{\top} \mathbf{p}$, we have:

$$
\frac{\partial \beta_{2}}{\partial \mathbf{x}} \mathbf{f}_{0}=\mathbf{e}_{3}^{\top} \mathbf{v}=\beta_{4}, \quad \frac{\partial \beta_{2}}{\partial \mathbf{x}} \mathbf{f}_{1 i}=0, \quad \frac{\partial \beta_{2}}{\partial \mathbf{x}} \mathbf{f}_{2 i}=0 .
$$

Then, for the span of $\boldsymbol{\beta}_{3} \triangleq \mathbf{C v}$, we have:

$$
\begin{aligned}
\frac{\partial \boldsymbol{\beta}_{3}}{\partial \mathbf{x}} \mathbf{f}_{0} & =-\lfloor\mathbf{C v}\rfloor \mathbf{b}_{g}+\mathbf{C g}-\mathbf{b}_{a} \\
& \triangleq-\left\lfloor\boldsymbol{\beta}_{3}\right\rfloor \boldsymbol{\beta}_{5}+\boldsymbol{\beta}_{6}-\boldsymbol{\beta}_{7} \\
\frac{\partial \boldsymbol{\beta}_{3}}{\partial \mathbf{x}} \mathbf{f}_{1 i} & =\lfloor\mathbf{C v}\rfloor \mathbf{e}_{i}=\left\lfloor\boldsymbol{\beta}_{3}\right\rfloor \mathbf{e}_{i}, \quad \frac{\partial \boldsymbol{\beta}_{3}}{\partial \mathbf{x}} \mathbf{f}_{2 i}=\mathbf{C} \mathbf{C}^{\top} \mathbf{e}_{i}=\mathbf{e}_{i},
\end{aligned}
$$

where the newly defined bases are $\boldsymbol{\beta}_{6} \triangleq \mathbf{C g}$ and $\boldsymbol{\beta}_{7} \triangleq \mathbf{b}_{a}$. 
Finally, for the span of $\beta_{4} \triangleq \mathbf{e}_{3}^{\top} \mathbf{v}$, we have:

$$
\begin{aligned}
\frac{\partial \beta_{4}}{\partial \mathbf{x}} \mathbf{f}_{0} & =\mathbf{e}_{3}^{\top}\left(\mathbf{g}-\mathbf{C}^{\top} \mathbf{b}_{a}\right)=\mathbf{e}_{3}^{\top} \mathbf{C}^{\top}\left(\mathbf{C g}-\mathbf{b}_{a}\right) \\
& =g^{-1} \boldsymbol{\beta}_{6}^{\top}\left(\boldsymbol{\beta}_{6}-\boldsymbol{\beta}_{7}\right) \\
\frac{\partial \beta_{4}}{\partial \mathbf{x}} \mathbf{f}_{1 i} & =0, \\
\frac{\partial \beta_{4}}{\partial \mathbf{x}} \mathbf{f}_{2 i} & =\mathbf{e}_{3}^{\top} \mathbf{C}^{\top} \mathbf{e}_{i}=\left(\mathbf{C e}_{3}\right)^{\top} \mathbf{e}_{i}=g^{-1}(\mathbf{C g})^{\top} \mathbf{e}_{i}=g^{-1} \boldsymbol{\beta}_{6}^{\top} \mathbf{e}_{i},
\end{aligned}
$$

where $g$ is the norm of the gravitational acceleration, g. At the next step, we repeat the same process of projecting the span of the newly defined basis functions $\boldsymbol{\beta}_{5}, \boldsymbol{\beta}_{6}$, and $\boldsymbol{\beta}_{7}$ on the process functions. Specifically, we have:

$$
\boldsymbol{\beta}_{5} \triangleq \mathbf{b}_{g} \text { : }
$$

$$
\begin{aligned}
& \frac{\partial \boldsymbol{\beta}_{5}}{\partial \mathbf{x}}=\left[\begin{array}{llllll}
\mathbf{0} & \mathbf{0} & \mathbf{0} & \mathbf{0} & \mathbf{I} & \mathbf{0}
\end{array}\right] \\
& \frac{\partial \boldsymbol{\beta}_{5}}{\partial \mathbf{x}} \mathbf{f}_{0}=\mathbf{0}, \quad \frac{\partial \boldsymbol{\beta}_{5}}{\partial \mathbf{x}} \mathbf{f}_{1 i}=\mathbf{0}, \quad \frac{\partial \boldsymbol{\beta}_{5}}{\partial \mathbf{x}} \mathbf{f}_{2 i}=\mathbf{0},
\end{aligned}
$$

$\boldsymbol{\beta}_{6} \triangleq \mathbf{C g}:$

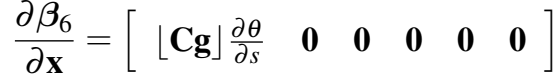

$$
\begin{aligned}
& \frac{\partial \boldsymbol{\beta}_{6}}{\partial \mathbf{x}} \mathbf{f}_{0}=-\lfloor\mathbf{C g}\rfloor \mathbf{b}_{g}=-\left\lfloor\boldsymbol{\beta}_{6}\right\rfloor \boldsymbol{\beta}_{5} \\
& \frac{\partial \boldsymbol{\beta}_{6}}{\partial \mathbf{x}} \mathbf{f}_{1 i}=\lfloor\mathbf{C g}\rfloor \mathbf{e}_{i}=\left\lfloor\boldsymbol{\beta}_{6}\right\rfloor \mathbf{e}_{i}, \quad \frac{\partial \boldsymbol{\beta}_{6}}{\partial \mathbf{x}} \mathbf{f}_{2 i}=\mathbf{0},
\end{aligned}
$$

$\boldsymbol{\beta}_{7} \triangleq \mathbf{b}_{a}$ :

$$
\begin{aligned}
& \frac{\partial \boldsymbol{\beta}_{7}}{\partial \mathbf{x}}=\left[\begin{array}{llllll}
\mathbf{0} & \mathbf{0} & \mathbf{0} & \mathbf{I} & \mathbf{0} & \mathbf{0}
\end{array}\right] \\
& \frac{\partial \boldsymbol{\beta}_{7}}{\partial \mathbf{x}} \mathbf{f}_{0}=\mathbf{0}, \quad \frac{\partial \boldsymbol{\beta}_{7}}{\partial \mathbf{x}} \mathbf{f}_{1 i}=\mathbf{0}, \quad \frac{\partial \boldsymbol{\beta}_{7}}{\partial \mathbf{x}} \mathbf{f}_{2 i}=\mathbf{0} .
\end{aligned}
$$

Since all the terms in the preceding projections are defined based on the existing basis functions (i.e., the second condition of Theorem 1 is satisfied), we have found a complete basis set.

The corresponding new process model for the defined bases can be described as:

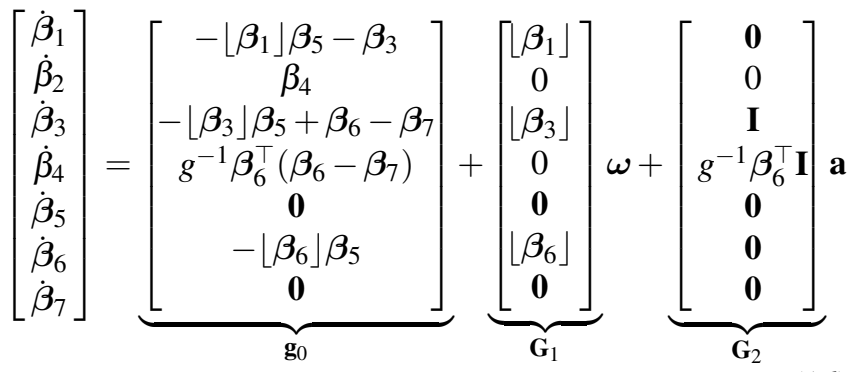

where

$$
\begin{aligned}
\mathbf{G}_{1} \boldsymbol{\omega} & =\mathbf{g}_{11} \omega_{1}+\mathbf{g}_{12} \omega_{2}+\mathbf{g}_{13} \omega_{3} \\
\mathbf{G}_{2} \mathbf{a} & =\mathbf{g}_{21} a_{1}+\mathbf{g}_{22} a_{2}+\mathbf{g}_{23} a_{3}
\end{aligned}
$$

Finally, the measurement equations can be expressed in terms of the basis functions as:

$$
\begin{aligned}
& \overline{\mathbf{h}}_{1}=\mathbf{B} \frac{1}{\beta_{2}} \boldsymbol{\beta}_{1} \\
& \bar{h}_{2}=\mathbf{e}_{3}^{\top} \boldsymbol{\beta}_{3}-\beta_{4}=0 .
\end{aligned}
$$

Equations (18) and (19) are used in Appendix I for constructing matrix $\Xi$ and showing that it is of full rank.

\section{B. Unobservable Directions of $\Omega$}

Based on Corollary 1 and given that the matrix $\Xi$ is of full rank (see also Appendix I) the unobservable directions of system (1) lie in the null space of matrix $\Omega$.

Stacking the derivatives of the basis functions with respect to the variable $\mathbf{x}$, the matrix $\Omega$ is

$$
\Omega=\left[\begin{array}{cccccc}
\left\lfloor\mathbf{C}\left(\mathbf{p}_{f}-\mathbf{p}\right)\right\rfloor \frac{\partial \theta}{\partial s} & \mathbf{0} & -\mathbf{C} & \mathbf{0} & \mathbf{0} & \mathbf{C} \\
\mathbf{0} & \mathbf{0} & \mathbf{e}_{3}^{\top} & \mathbf{0} & \mathbf{0} & \mathbf{0} \\
\lfloor\mathbf{C v}\rfloor \frac{\partial \theta}{\partial s} & \mathbf{C} & \mathbf{0} & \mathbf{0} & \mathbf{0} & \mathbf{0} \\
\mathbf{0} & \mathbf{e}_{3}^{\top} & \mathbf{0} & \mathbf{0} & \mathbf{0} & \mathbf{0} \\
\mathbf{0} & \mathbf{0} & \mathbf{0} & \mathbf{0} & \mathbf{I} & \mathbf{0} \\
\mathbf{C g}\rfloor \frac{\partial \theta}{\partial s} & \mathbf{0} & \mathbf{0} & \mathbf{0} & \mathbf{0} & \mathbf{0} \\
\mathbf{0} & \mathbf{0} & \mathbf{0} & \mathbf{I} & \mathbf{0} & \mathbf{0}
\end{array}\right] .
$$

To describe the null space of matrix $\Omega$, we need to find a matrix

$$
\mathbf{A}=\left[\mathbf{A}_{1}^{\top}, \mathbf{A}_{2}^{\top}, \mathbf{A}_{3}^{\top}, \mathbf{A}_{4}^{\top}, \mathbf{A}_{5}^{\top}, \mathbf{A}_{6}^{\top}\right]^{\top} \neq \mathbf{0},
$$

such that

$$
\Omega \mathbf{A}=\mathbf{0},
$$

in other words, $\mathbf{A}$ spans the full null space of $\Omega$. From (21), we have: 6

- Multiplying the fifth and the seventh block rows of $\Omega$ with $\mathbf{A}$, we get $\mathbf{A}_{4}=\mathbf{A}_{5}=\mathbf{0}$.

- Multiplying the sixth block row of $\Omega$ with $\mathbf{A}$, we have $\lfloor\mathbf{C g}\rfloor \frac{\partial \theta}{\partial s} \mathbf{A}_{1}=\mathbf{0}$, which implies that either $\mathbf{A}_{1}=\mathbf{0}$ or $\mathbf{A}_{1}=\frac{\partial s}{\partial \theta} \mathbf{C g}$.

1) If $\mathbf{A}_{1}=\mathbf{0}$, then from the third block row of $\Omega \mathbf{A}=\mathbf{0}$ we have $\mathbf{C A}_{2}=\mathbf{0} \Rightarrow \mathbf{A}_{2}=\mathbf{0}$, since $\mathbf{C}$ is a rotation matrix (full rank). For the first block row of $\Omega \mathbf{A}$ to be zero, we have $\mathbf{A}_{3}=\mathbf{A}_{6}$. Finally, from the second block row of $\Omega \mathbf{A}=\mathbf{0}$, we get $\mathbf{e}_{3}^{\top} \mathbf{A}_{3}=\mathbf{0}$. This implies that $\mathbf{A}_{3}$ is spanned by $\mathbf{e}_{1}$ and $\mathbf{e}_{2}$, i.e.,

$$
\mathbf{A}_{3}=\mathbf{A}_{6}=\left[\begin{array}{c}
\mathbf{I}_{2} \\
\mathbf{0}_{1 \times 2}
\end{array}\right] \text {. }
$$

2) If $\mathbf{A}_{1}=\frac{\partial s}{\partial \theta} \mathbf{C g}$, from the first block row of $\Omega \mathbf{A}=\mathbf{0}$, we have $\mathbf{A}_{3}=-\lfloor\mathbf{p}\rfloor \mathbf{g}$ and $\mathbf{A}_{6}=-\left\lfloor\mathbf{p}_{f}\right\rfloor \mathbf{g}$. Then, from its third block row, we get $\mathbf{A}_{2}=-\lfloor\mathbf{v}\rfloor \mathbf{g}$.

Hence, the system's unobservable directions are spanned by

$$
\mathbf{A}=\left[\begin{array}{cc}
\mathbf{0}_{3 \times 2} & \frac{\partial s}{\partial \theta} \mathbf{C g} \\
\mathbf{0}_{3 \times 2} & -\lfloor\mathbf{v}\rfloor \mathbf{g} \\
{\left[\begin{array}{c}
\mathbf{I}_{2} \\
\mathbf{0}_{1 \times 2}
\end{array}\right]} & -\lfloor\mathbf{p}\rfloor \mathbf{g} \\
\mathbf{0}_{3 \times 2} & \mathbf{0}_{3 \times 1} \\
\mathbf{0}_{3 \times 2} & \mathbf{0}_{3 \times 1} \\
{\left[\begin{array}{c}
\mathbf{I}_{2} \\
\mathbf{0}_{1 \times 2}
\end{array}\right]} & -\left\lfloor\mathbf{p}_{f}\right\rfloor \mathbf{g}
\end{array}\right] .
$$

\footnotetext{
${ }^{6}$ The choice of the null-space bases is done so that meaningful physical interpretations can be made.
} 


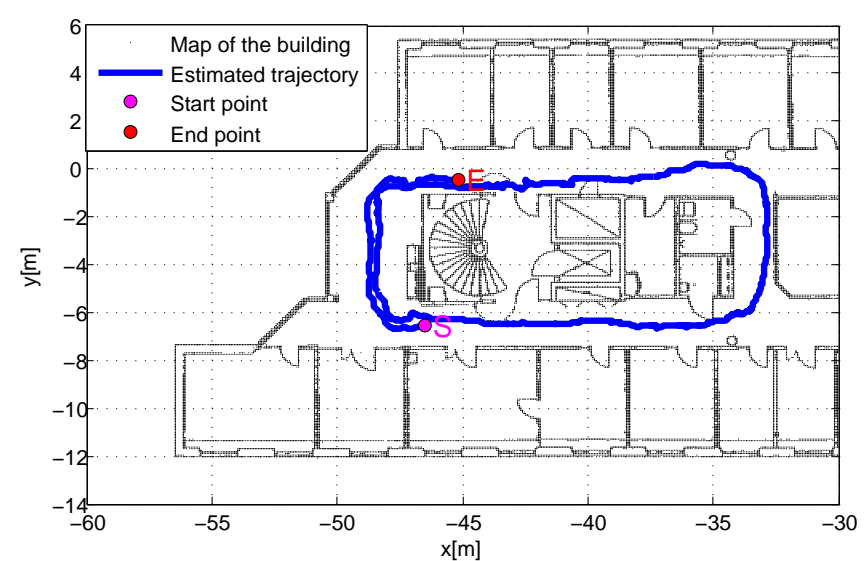

(a) Estimated position along the $x$ and $y$ axes

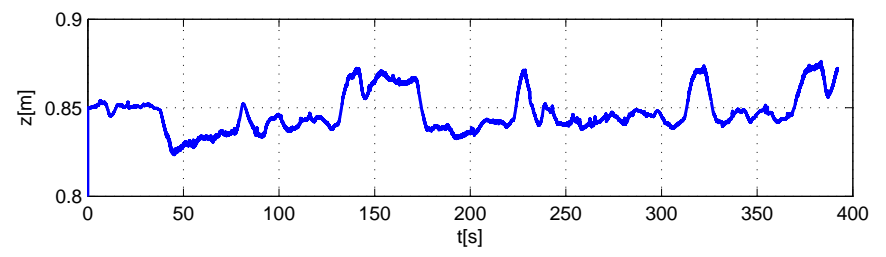

(b) Estimated height along the $z$ axis

Fig. 1: (a) The estimated trajectory of the IMU-camera along the $x-y$ plane overlaid on the map of the test environment. (b) The estimated height of the system; the IMU-camera rig was mounted at a height of $85 \mathrm{~cm}$.

These unobservable directions correspond to the system's (IMU-camera pair) and landmark's motion parallel to the $x-y$ plane (first two columns of $\mathbf{A}$ ), and their rotation about the gravity vector (third column of $\mathbf{A}$ ). It should be noted that adding more point features does not change the observable modes of the system. The extension of this observability analysis to the case of multiple point features is described in [13].

\section{EXPERIMENTAL RESUlts}

The key findings of the observability analysis for the DLCVINS are validated using the method described in [1], where the camera's optical axis is assumed to be orthogonal to the ground plane.

In our experiments, we employ an AVT Guppy monochrome camera with sampling rate of $10 \mathrm{~Hz}$ that is rigidly mounted on top of a MicroStrain 3DMGX2 IMU which has sampling rate $250 \mathrm{~Hz}$. The camera intrinsic calibration is done using [14] and the IMU-camera calibration parameters are estimated from [15]. The MATLAB Computer Vision Toolbox implementation of SURF is used for the feature extraction and matching.

An example of the estimated trajectory overlaid on the map of the test environment is plotted in Fig. 1(a). The path length was about $50 \mathrm{~m}$ and it was travelled within 400 seconds. For the experiment, the IMU-camera sensor fusion system was mounted on a cart $85 \mathrm{~cm}$ off the ground such that the camera's optical axis was approximately orthogonal to the ground plane. Additionally, the estimated height of the mobile system is plotted in Fig. 1(b). Note that the
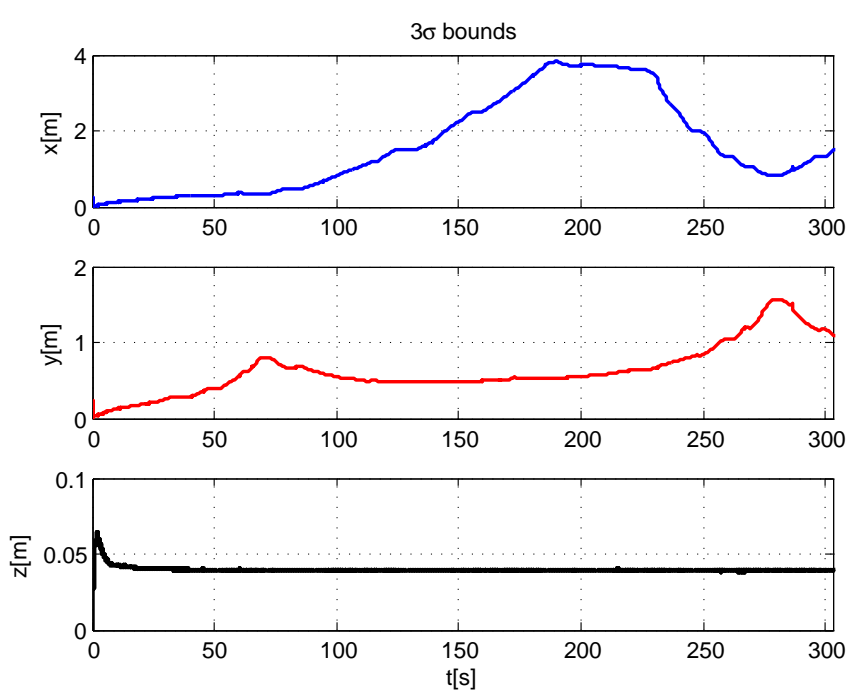

(a) Position
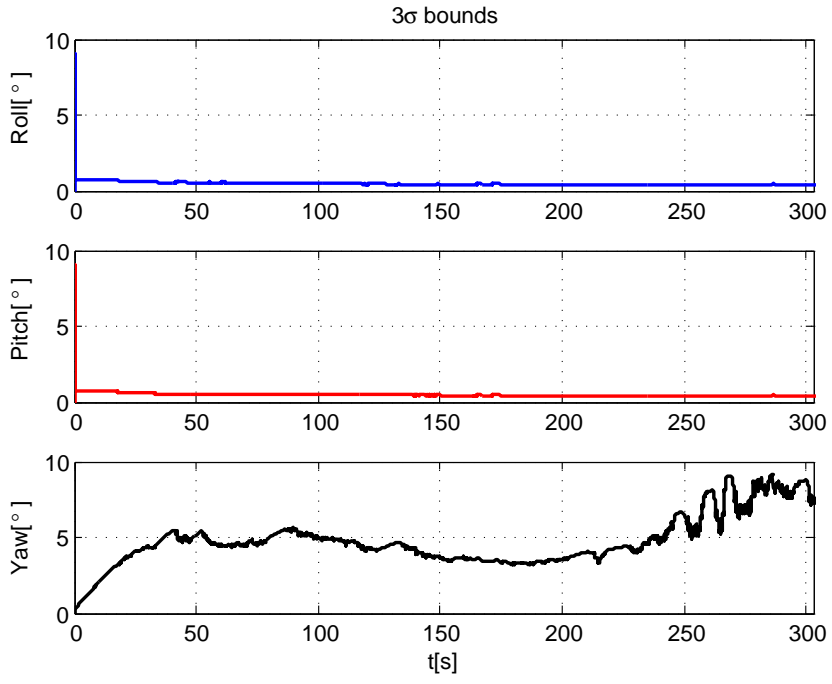

(b) Attitude
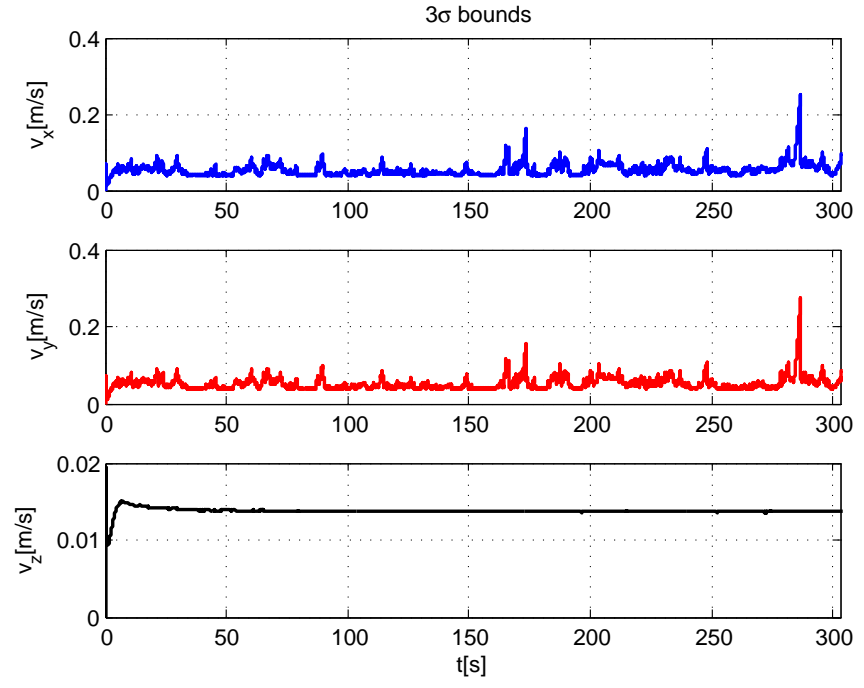

(c) Velocity

Fig. 2: The $3 \sigma$ bounds for the error in position (a), attitude (b), and velocity (c). The $\sigma$ values are computed as the square roots of the corresponding diagonal elements of the states' error covariance matrix. 


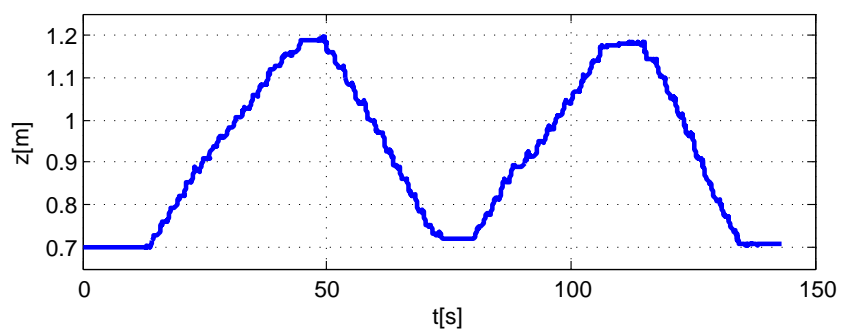

Fig. 3: Estimated height along the $z$ axis.

estimated height is diverging from the approximated true value only briefly (due to the lack of correctly detected and matched point features), while for most of the time the system is capable of error correction that drastically reduces the estimated height error.

To better quantify the estimation uncertainty, the $3 \sigma$ bounds for the error in the camera position, orientation, and velocity along the three axes are shown in Fig. 2. The $\sigma$ values are the square roots of the corresponding diagonal elements of the states' error covariance matrix. As depicted in Fig. 2(a), the uncertainty of the position along the $x$ and $y$ axes grows slowly since the system has no access to absolute position information. In contrast, the uncertainty ( $3 \sigma$ bound) along the $z$ axis remains constant, which confirms the findings of our observability analysis (i.e., the height is observable). The results shown in Fig. 2(b) and Fig. 2(c) are also inline with our analysis; the velocity, roll, and pitch are observable while the yaw is unobservable.

Furthermore, the precision of the height estimation was examined by placing the IMU-camera on a test table where the system was only moved up and lowered down parallel to the gravity. Fig. 3 shows the estimated height for this experiment. The system was lifted up and down to approximately the same values of height by a step motor for about 140 seconds. The final error in the $x-y$ plane is approximately $0.2 \mathrm{~cm}$.

\section{CONCLUSION}

In this paper, we have studied the observability properties of a VINS in which the camera only observes point features on the ground and its optical axis is aligned with gravity. Compared to general VINS, where features are not constrained to be on a plane, we have proved that in our system the vertical distance of the camera with respect to a global frame is observable. In the analysis, the full INS state variables, including position, velocity, and rotation of the camera in addition to the IMU biases, are considered. Furthermore, we have derived all the observable and unobservable directions of the system using only one point feature as the camera measurement. Finally, we have experimentally verified the key findings of our observability analysis.

\section{REFERENCES}

[1] G. Panahandeh, D. Zachariah, and M. Jansson, "Exploiting ground plane constraints for visual-inertial navigation," in Proc. IEEE-ION Position Location and Navigation Symposium, Myrtle Beach, South Carolina, Apr. 23-26, 2012, pp. 527-534.
[2] A. I. Mourikis, N. Trawny, S. I. Roumeliotis, A. E. Johnson, A. Ansar, and L. Matthies, "Vision-aided inertial navigation for spacecraft entry, descent, and landing," IEEE Trans. on Robotics, vol. 25, no. 2, pp. 264-280, 2009.

[3] F. M. Mirzaei and S. I. Roumeliotis, "A kalman filter-based algorithm for imu-camera calibration: Observability analysis and performance evaluation," IEEE Trans. on Robotics, vol. 24, no. 5, pp. 1143-1156, 2008.

[4] J. Kelly and G. S. Sukhatme, "Visual-inertial sensor fusion: Localization, mapping and sensor-to-sensor self-calibration," Int. Journal of Robotics Research, vol. 30, no. 1, pp. 56-79, 2011.

[5] J. A. Hesch, D. G. Kottas, S. L. Bowman, and S. I. Roumeliotis, "Towards consistent vision-aided inertial navigation," in Proc. 10th Int. Workshop on the Algorithmic Foundations of Robotics, Cambridge, MA, Jun. 13-15, 2012.

[6] M. Li and A. I. Mourikis, "Improving the accuracy of ekf-based visual-inertial odometry," in Proc. IEEE Int. Conf. on Robotics and Automation (ICRA), Saint Paul, Minnesota, May 14-18, 2012, pp. 828835.

[7] E. S Jones and S. Soatto, "Visual-inertial navigation, mapping and localization: A scalable real-time causal approach," Int. Journal of Robotics Research, vol. 30, no. 4, pp. 407-430, 2011.

[8] A. Martinelli, "Vision and imu data fusion: Closed-form solutions for attitude, speed, absolute scale, and bias determination," IEEE Trans. on Robotics, vol. 28, no. 1, pp. 44-60, 2012.

[9] C. Troiani and A. Martinelli, "Vision-aided inertial navigation using virtual features," in Proc. IEEE/RSJ Int. Conf. on Intelligent Robots and Systems (IROS), Vilamoura, Algarve, Portugal, Oct. 7-12, 2012, pp. 4828-4834.

[10] C. X. Guo and S. I. Roumeliotis, "IMU-RGBD camera extrinsic calibration: Observability analysis and consistency improvement," in Proc. IEEE Int. Conf. on Robotics and Automation (ICRA), Karlsruhe, Germany, May 6-10, 2013, pp. 2920-2927.

[11] R. Hermann and A. Krener, "Nonlinear controllability and observability," IEEE Trans. on Automatic Control, vol. 22, no. 4, pp. 728-740, 1977.

[12] M. D. Shuster, "A survey of attitude representations," Astronautical Sciences, vol. 41, no. 4, pp. 439-517, 1993.

[13] G. Panahandeh, C. X. Guo, M. Jansson, and S. I. Roumeliotis, "Observability analysis of a vision-aided inertial navigation system using planar features on the ground: Supplemental material," in Technical Report, http://urn.kb.se/resolve?urn=urn:nbn: se:kth:diva-124655, 2013.

[14] J. Y. Bouguet, "Camera calibration toolbox," Computational vision at Caltech, www.vision.caltech.edu/bouguetj/, last accessed Mar. 2012.

[15] G. Panahandeh and M. Jansson, "IMU-camera self-calibration using planar mirror reflection," in Proc. IEEE Int. Conf. on Indoor Positioning and Indoor Navigation (IPIN), Guimares, Portugal, Sep. 21-23, 2011, pp. 1-7. 


\section{APPENDIX I}

To prove that matrix $\Xi$ is of full column rank, it suffices to show that a subset of its rows, whose dimension is the same or larger than the number of columns, is linearly independent. To show this, we construct a sub-matrix of $\Xi$ by selecting the minimum number of Lie derivative gradients of the measurement functions (18) and (19) that leads to a full-column-rank matrix. Note that, finding the proper Lie derivatives, which lead to the minimum sub-matrix dimension, is quiet challenging. Empirically, one can seek to find the directions of the state space along which the gradients of each of the candidate Lie derivatives provides new information. In the following, we compute only those Lie derivatives of $\overline{\mathbf{h}}_{1}$ and $\bar{h}_{2}$ whose spans are used to prove that $\Xi$ is of full column rank.

- The zeroth-order Lie derivatives of the measurement functions are:

$$
\begin{aligned}
\mathfrak{L}^{0} \overline{\mathbf{h}}_{1} & =\mathbf{B} \frac{1}{\beta_{2}} \boldsymbol{\beta}_{1}, \\
\mathfrak{L}^{0} \bar{h}_{2} & =\mathbf{e}_{3}^{\top} \boldsymbol{\beta}_{3}-\beta_{4} .
\end{aligned}
$$

Then, their gradient (i.e., the span) of each zeroth order Lie derivative is

$$
\begin{aligned}
\nabla \mathfrak{L}^{0} \overline{\mathbf{h}}_{1} & =\frac{\partial \overline{\mathbf{h}}_{1}}{\partial \boldsymbol{\beta}}=\mathbf{B}\left[\begin{array}{lllllll}
\frac{1}{\beta_{2}} \mathbf{I} & -\frac{\boldsymbol{\beta}_{1}}{\beta_{2}^{2}} & \mathbf{0} & \mathbf{0} & \mathbf{0} & \mathbf{0} & \mathbf{0}
\end{array}\right], \\
\nabla \mathfrak{L}^{0} h_{2} & =\frac{\partial \overline{\mathbf{h}}_{2}}{\partial \boldsymbol{\beta}}=\left[\begin{array}{lllllll}
\mathbf{0} & 0 & \mathbf{e}_{3}^{\top} & -1 & \mathbf{0} & \mathbf{0} & \mathbf{0}
\end{array}\right] .
\end{aligned}
$$

- The first-order Lie derivatives of $\overline{\mathbf{h}}_{1}$ with respect to $\mathbf{g}_{0}$, and $\mathbf{g}_{1 i}$ are computed, respectively, as

$$
\begin{aligned}
\mathfrak{L}_{\mathbf{g}_{0}}^{1} \overline{\mathbf{h}}_{1}=\nabla \mathfrak{L}^{0} \overline{\mathbf{h}}_{1} \cdot \mathbf{g}_{0}=\mathbf{B}\left(\frac{1}{\beta_{2}}\left(-\left\lfloor\boldsymbol{\beta}_{1}\right\rfloor \boldsymbol{\beta}_{5}-\boldsymbol{\beta}_{3}\right)-\frac{\beta_{4}}{\beta_{2}^{2}} \boldsymbol{\beta}_{1}\right), \\
\mathfrak{L}_{\mathbf{g}_{i i}}^{1} \overline{\mathbf{h}}_{1}=\nabla \mathfrak{L}^{0} \overline{\mathbf{h}}_{1} \cdot \mathbf{g}_{1 i}=\mathbf{B} \frac{1}{\beta_{2}}\left\lfloor\boldsymbol{\beta}_{1}\right\rfloor \mathbf{e}_{i},
\end{aligned}
$$

while their corresponding gradients are given by

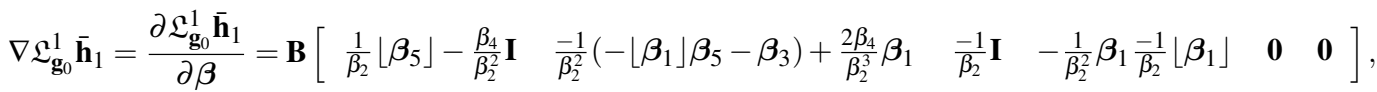

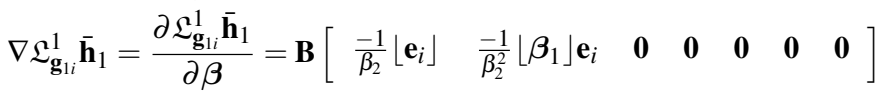

- The second-order Lie derivatives and their corresponding gradients: ${ }^{7}$

$$
\begin{aligned}
& \mathfrak{L}_{\mathbf{g}_{0} \mathbf{g}_{1 i}}^{2} \overline{\mathbf{h}}_{1}=\nabla \mathfrak{L}_{\mathbf{g}_{0}}^{1} \overline{\mathbf{h}}_{1} \cdot \mathbf{g}_{1 i}=\mathbf{B}\left(\left(\frac{1}{\beta_{2}}\left\lfloor\boldsymbol{\beta}_{5}\right\rfloor-\frac{\beta_{4}}{\beta_{2}^{2}} \mathbf{I}\right)\left\lfloor\boldsymbol{\beta}_{1}\right\rfloor \mathbf{e}_{i}+\frac{-1}{\beta_{2}}\left\lfloor\boldsymbol{\beta}_{3}\right\rfloor \mathbf{e}_{i}\right), \\
& \mathfrak{L}_{\mathbf{g}_{1} \mathbf{g}_{0}}^{2} \overline{\mathbf{h}}_{1}=\nabla \mathfrak{L}_{\mathbf{g}_{1 i}}^{1} \overline{\mathbf{h}}_{1} \cdot \mathbf{g}_{0}=\mathbf{B}\left(\frac{-1}{\beta_{2}}\left\lfloor\mathbf{e}_{i}\right\rfloor\left(-\left\lfloor\boldsymbol{\beta}_{1}\right\rfloor \boldsymbol{\beta}_{5}-\boldsymbol{\beta}_{3}\right)+\frac{-\beta_{4}}{\beta_{2}^{2}}\left\lfloor\boldsymbol{\beta}_{1}\right\rfloor \mathbf{e}_{i}\right),
\end{aligned}
$$

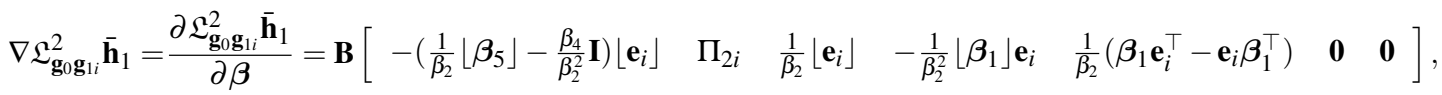

$$
\begin{aligned}
& \nabla \mathfrak{L}_{\mathbf{g}_{i} \mathbf{g}_{0}}^{2} \overline{\mathbf{h}}_{1}=\frac{\partial \mathfrak{L}_{\mathbf{g}_{1} \mathbf{g}_{0}}^{2} \overline{\mathbf{h}}_{1}}{\partial \boldsymbol{\beta}}=\mathbf{B}\left[\frac{-1}{\beta_{2}}\left\lfloor\mathbf{e}_{i}\right\rfloor\left\lfloor\boldsymbol{\beta}_{5}\right\rfloor+\frac{\beta_{4}}{\beta_{2}^{2}}\left\lfloor\mathbf{e}_{i}\right\rfloor \quad \frac{-1}{\beta_{2}^{2}}\left\lfloor\mathbf{e}_{i}\right\rfloor\left(\left\lfloor\boldsymbol{\beta}_{1}\right\rfloor \boldsymbol{\beta}_{5}+\boldsymbol{\beta}_{3}\right)+\frac{2 \beta_{4}}{\beta_{2}^{3}}\left\lfloor\boldsymbol{\beta}_{1}\right\rfloor \mathbf{e}_{i} \quad \frac{1}{\beta_{2}}\left\lfloor\mathbf{e}_{i}\right\rfloor \frac{-1}{\beta_{2}^{2}}\left\lfloor\boldsymbol{\beta}_{1}\right\rfloor \mathbf{e}_{i} \quad \frac{1}{\beta_{2}}\left\lfloor\mathbf{e}_{i}\right\rfloor\left\lfloor\boldsymbol{\beta}_{1}\right\rfloor \quad \mathbf{0} \quad \mathbf{0}\right] .
\end{aligned}
$$

- The third-order Lie derivatives and their corresponding gradients are:

$$
\begin{aligned}
& \mathfrak{L}_{\mathbf{g}_{0} \mathbf{g}_{i 1} \mathbf{g}_{0}}^{\mathbf{g}_{1}} \overline{\mathbf{h}}_{1}=\nabla \mathfrak{L}_{\mathbf{g}_{0} \mathbf{g}_{1 i}}^{2} \overline{\mathbf{h}}_{1} \cdot \mathbf{g}_{0}=\mathbf{B}\left(-\left(\frac{1}{\beta_{2}}\left\lfloor\boldsymbol{\beta}_{5}\right\rfloor-\frac{\beta_{4}}{\beta_{2}^{2}} \mathbf{I}\right)\left\lfloor\mathbf{e}_{i}\right\rfloor\left(-\left\lfloor\boldsymbol{\beta}_{1}\right\rfloor \boldsymbol{\beta}_{5}-\boldsymbol{\beta}_{3}\right)+\Pi_{2 i} \beta_{4}+\frac{1}{\beta_{2}}\left\lfloor\mathbf{e}_{i}\right\rfloor\left(-\left\lfloor\boldsymbol{\beta}_{3}\right\rfloor \boldsymbol{\beta}_{5}+\boldsymbol{\beta}_{6}-\boldsymbol{\beta}_{7}\right)-\right. \\
& \left.\frac{g^{-1}}{\beta_{2}^{2}}\left\lfloor\boldsymbol{\beta}_{1}\right\rfloor \mathbf{e}_{i}\left(\boldsymbol{\beta}_{6}^{\top}\left(\boldsymbol{\beta}_{6}-\boldsymbol{\beta}_{7}\right)\right)\right), \\
& \mathfrak{L}_{\mathbf{g}_{0} \mathbf{g}_{1 i} \mathbf{g}_{2 j}}^{3} \overline{\mathbf{h}}_{1}=\nabla \mathfrak{L}_{\mathbf{g}_{0} \mathbf{g}_{1 i}}^{2} \overline{\mathbf{h}}_{1} \cdot \mathbf{g}_{2 j}=\frac{1}{\beta_{2}}\left\lfloor\mathbf{e}_{i}\right\rfloor \mathbf{e}_{j}-\frac{g^{-1}}{\beta_{2}^{2}}\left\lfloor\boldsymbol{\beta}_{1}\right\rfloor \mathbf{e}_{i} \boldsymbol{\beta}_{6}^{\top} \mathbf{e}_{j},
\end{aligned}
$$

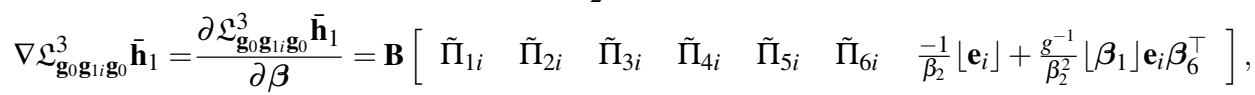

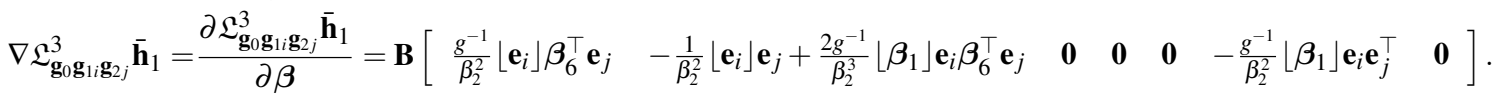

${ }^{7} \Pi_{i}$ and $\tilde{\Pi}_{i j}$ will be removed later on by Gaussian elimination, thus we do not show their explicit expressions here. 
Stacking together the computed spans of the Lie derivatives, a subset of the observability matrix $\Xi$ is constructed as:

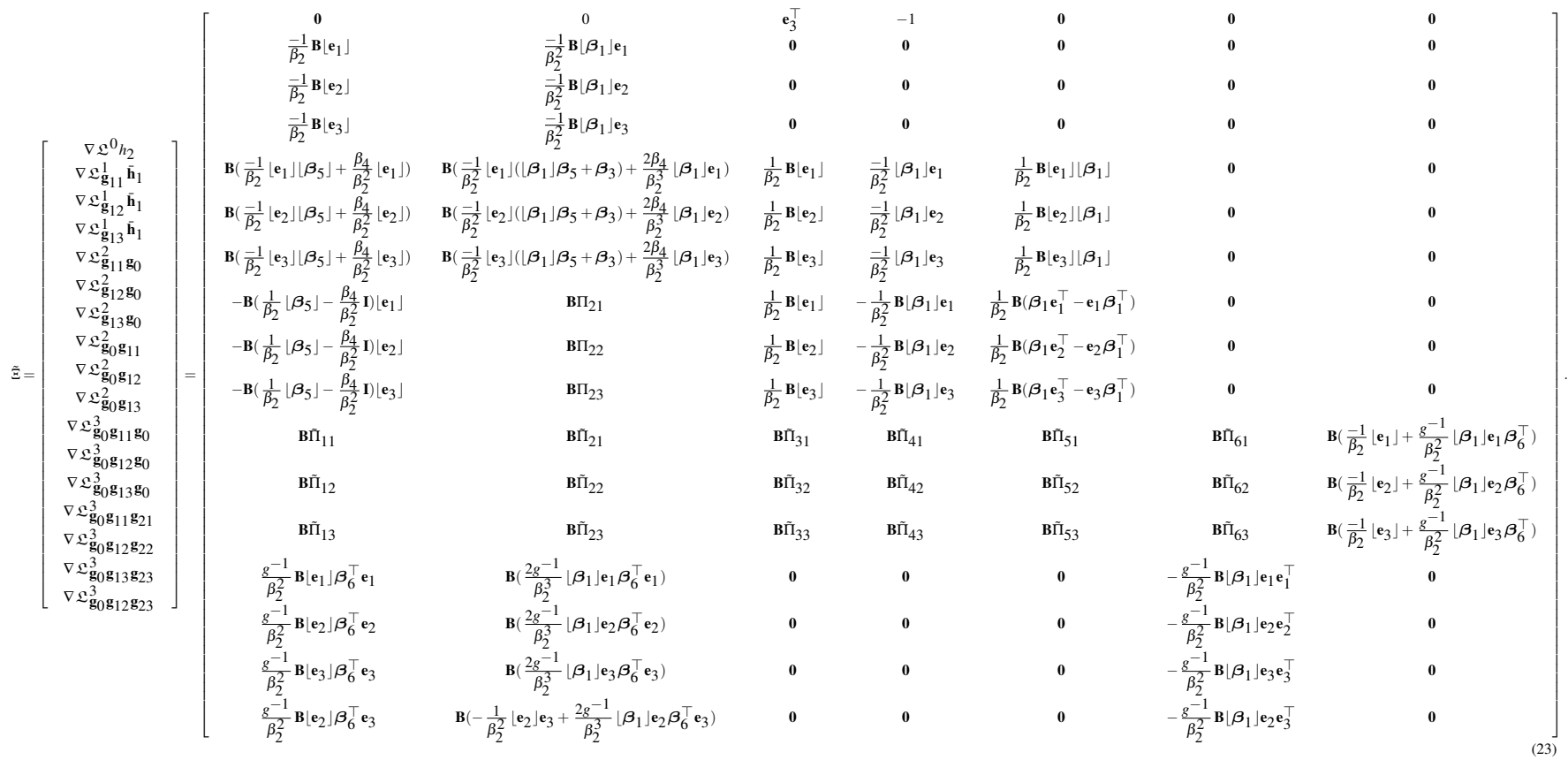

To show that $\Xi$ is of full rank, we use Gaussian elimination. The Gaussian elimination is performed based on the knowledge that the following matrices are of full column rank, which they clearly are as long as $u$ and $v,(8)$, are not both zero.

$$
\begin{gathered}
\Upsilon=\frac{1}{\beta_{2}}\left[\begin{array}{l}
\mathbf{B}\left\lfloor\mathbf{e}_{1}\right\rfloor \\
\mathbf{B}\left\lfloor\mathbf{e}_{2}\right\rfloor \\
\mathbf{B}\left\lfloor\mathbf{e}_{3}\right\rfloor
\end{array}\right]=\frac{1}{\beta_{2}}\left[\begin{array}{ccc}
0 & 0 & 0 \\
0 & 0 & -1 \\
0 & 0 & 1 \\
0 & 0 & 0 \\
0 & -1 & 0 \\
1 & 0 & 0
\end{array}\right], \quad \hat{\Upsilon}=\frac{-g^{-1}}{\beta_{2}^{2}}\left[\begin{array}{l}
\mathbf{B}\left\lfloor\boldsymbol{\beta}_{1}\right\rfloor \mathbf{e}_{1} \mathbf{e}_{1}^{\top} \\
\mathbf{B}\left\lfloor\boldsymbol{\beta}_{1}\right\rfloor \mathbf{e}_{2} \mathbf{e}_{2}^{\top} \\
\mathbf{B}\left\lfloor\boldsymbol{\beta}_{1}\right\rfloor \mathbf{e}_{3} \mathbf{e}_{3}^{\top}
\end{array}\right]=\frac{-g^{-1}}{\beta_{2}}\left[\begin{array}{ccc}
0 & 0 & 0 \\
1 & 0 & 0 \\
0 & -1 & 0 \\
0 & 0 & 0 \\
0 & 0 & v \\
0 & 0 & -u
\end{array}\right], \\
\tilde{\Upsilon}=\frac{-1}{\beta_{2}}\left[\begin{array}{l}
\mathbf{B}\left\lfloor\boldsymbol{\beta}_{1}\right\rfloor\left\lfloor\mathbf{e}_{1}\right\rfloor \\
\mathbf{B}\left\lfloor\boldsymbol{\beta}_{1}\right\rfloor\left\lfloor\mathbf{e}_{2}\right\rfloor \\
\mathbf{B}\left\lfloor\boldsymbol{\beta}_{1}\right\rfloor\left\lfloor\mathbf{e}_{3}\right\rfloor
\end{array}\right]=\left[\begin{array}{ccc}
0 & v & 1 \\
0 & -u & 0 \\
-v & 0 & 0 \\
u & 0 & 1 \\
-1 & 0 & 0 \\
0 & -1 & 0
\end{array}\right], \quad \hat{\Upsilon}=\left[\begin{array}{l}
\frac{-1}{\beta_{2}} \mathbf{B}\left\lfloor\mathbf{e}_{1}\right\rfloor+\frac{g^{-1}}{\beta_{2}^{2}} \mathbf{B}\left\lfloor\boldsymbol{\beta}_{1}\right\rfloor \mathbf{e}_{1} \boldsymbol{\beta}_{6}^{\top} \\
\frac{-1}{\beta_{2}} \mathbf{B}\left\lfloor\mathbf{e}_{2}\right\rfloor+\frac{g^{-1}}{\beta_{2}^{2}} \mathbf{B}\left\lfloor\boldsymbol{\beta}_{1}\right\rfloor \mathbf{e}_{2} \boldsymbol{\beta}_{6}^{\top} \\
\frac{-1}{\beta_{2}} \mathbf{B}\left\lfloor\mathbf{e}_{3}\right\rfloor+\frac{g^{-1}}{\beta_{2}^{2}} \mathbf{B}\left\lfloor\boldsymbol{\beta}_{1}\right\rfloor \mathbf{e}_{3} \boldsymbol{\beta}_{6}^{\top}
\end{array}\right]=\frac{1}{\beta_{2}}\left[\begin{array}{ccc}
0 & 0 & 0 \\
0 & 0 & 2 \\
0 & 0 & -2 \\
0 & 0 & 0 \\
0 & 1 & v \\
-1 & 0 & u
\end{array}\right], \\
\check{\Upsilon}=\mathbf{B}\left\lfloor\mathbf{e}_{2}\right\rfloor \mathbf{e}_{3}=[1 \quad 0]^{\top} .
\end{gathered}
$$

Performing Gaussian elimination by column-row operations on (23), yields:

$$
\bar{\Xi}=\left[\begin{array}{lllllll}
\mathbf{0} & 0 & \mathbf{0} & 2 & \mathbf{0} & \mathbf{0} & \mathbf{0} \\
\mathbf{I} & \mathbf{0} & \mathbf{0} & \mathbf{0} & \mathbf{0} & \mathbf{0} & \mathbf{0} \\
\mathbf{0} & \mathbf{0} & \mathbf{I} & \mathbf{0} & \mathbf{0} & \mathbf{0} & \mathbf{0} \\
\mathbf{0} & \mathbf{0} & \mathbf{0} & \mathbf{0} & \mathbf{I} & \mathbf{0} & \mathbf{0} \\
\mathbf{0} & \mathbf{0} & \mathbf{0} & \mathbf{0} & \mathbf{0} & \mathbf{0} & \mathbf{I} \\
\mathbf{0} & \mathbf{0} & \mathbf{0} & \mathbf{0} & \mathbf{0} & \mathbf{I} & \mathbf{0} \\
\mathbf{0} & 1 & \mathbf{0} & 0 & \mathbf{0} & \mathbf{0} & \mathbf{0}
\end{array}\right],
$$

which clearly is a full column rank matrix. Hence, the system described by (5) is observable and our defined basis functions are the system's observable modes. 